\title{
Correlación: vocación profesional - motivación por el estudio del alumno de educación superior universitaria, Amazonas, 2015
}

\section{Correlation: professional vocation - motivation for the study student university higher education, Amazonas, 2015}

\author{
José Darwin Farje-Escobedo', Mirtha Chuquizuta Culquimboz ${ }^{2}$
}

\section{RESUMEN}

El objetivo del trabajo fue determinar el nivel de correlación entre la vocación profesional y la motivación por el estudio de los alumnos de la escuela profesional de educación intercultural bilingüe y de la escuela profesional de educación, Facultad de Ciencias Sociales y Humanidades de la Universidad Nacional Toribio Rodríguez de Mendoza de Amazonas, 2015. La investigación fue de tipo descriptivo correlacional (Hernández, Fernández y Baptista, 2010). El objeto de estudio, estudiante universitario. La población estuvo conformado por 666 estudiantes, la muestra por 243 estudiantes de diferentes especialidades. Se utilizó como técnica la observación, como instrumento, ficha de encuesta, en escala tipo likert, los métodos utilizados fueron: inductivo, deductivo, analítico y sintético. Los resultados evidencian que existe una correlación directa alta entre la vocación profesional y la motivación por el estudio del alumno que estudia educación; un 50\% de estudiantes dice estar de acuerdo y totalmente de acuerdo con su vocación profesional para sus estudios de educador y un $47 \%$ dice estar motivado para el estudio en la profesión de educación. La correlación de variables en función al género también es directa alta, dado que el 49 y $46 \%$ de estudiantes mujeres tienen vocación profesional y están motivados por el estudio, respectivamente.

Palabras clave: vocación profesional, motivación por el estudio.

\begin{abstract}
The aim of the study was to determine the level of correlation between professional vocation and motivation for the study of students in vocational school of intercultural bilingual education and vocational school education, from the Faculty of Social Sciences and Humanities, National Toribio University Rodríguez de Mendoza Amazonas, 2015. The research was descriptive correlational kind (Hernandez, Fernández y Baptista, 2010). The study objet are college students. The population was made up for 666 students, the sample for 243 students of different specialities. The technique used was observation, and as an instrument, record of survey, Likert scale kind. The methods used were inductive and deductive. Correlational contrastation was calculated using Pearson's test. The results show that there is a direct correlation between professional vocation and motivation for the study of student studying education ( 0.838 bilateral, Pearson's Test). 50\% of students say they agree and fully agree with his professional vocation, and $47 \%$ say they are motivated to their study. The correlation of variables according to gender is also direct high, as 49 and $46 \%$ of women students, and 50 and $49 \%$ of man students, they have professional vocation and are motivated by the study, respectively.
\end{abstract}

Keywords: professional vocation, motivation for the study.

\footnotetext{
${ }^{1}$ Licenciado en Educación, Dr. en ciencias ambientales, Docente Asociado de la Universidad Nacional Toribio Rodríguez de Mendoza de Amazonas,josfarje@gmail.com

${ }^{2}$ Licenciada en Educación, Mg. en docencia y gestión universitaria, Docente Ordinaria de Educación Básica

Regular,mithachuquizuta@hotmail.com
} 


\section{INTRODUCCIÓN}

La sociedad de nuestro tiempo asiste a claros desafíos que se dan en los aspectos social, cultural, político, ambiental, que demandan mayores habilidades, capacidades y competencias a los individuos y sobre todo al segmento de profesionales. Por tanto, la vocación profesional concebida como el deseo de emprender una carrera, profesión o cualquier otro oficio juega un papel importante a la hora de elegir una opción para la profesionalización. Los estudiantes de las carreras profesionales de educación, al parecer, tienen una escasa vocación por su profesión. Al respecto, el Mineduc de Chile (2014: 9), considera que elegir la carrera profesional implica varios factores a considerar como, intereses y capacidades, por otro lado, las expectativas laborales.

La desmotivación de los estudiantes de educación y otras carreras, está asociada a diferentes factores como señala Díaz y Martins (1982: 18), en la educación profesional de pedagogía y otras carreras, en su mayoría, los docentes son autodidactas y sólo algunos tienen la oportunidad de participar en cursos de didáctica. (...) por esa falta de preparación para la docencia se muestran inseguros en su relación con los alumnos. Para mantener su autoridad y la imagen de sí mismos, recurren a actitudes protectoras, tales como comunicaciones muy formales con los alumnos, desmedido nivel de exigencia en las pruebas y empleo de la ironía y el sarcasmo para reducir a los rebeldes.

En el Perú, el Minedu planteó políticas orientadas a mejorar la educación superior, al respecto Ayzanoa (2003:52) sostiene que en las últimas décadas se han realizado diferentes esfuerzos orientados a mejorar la calidad de educación en todos los niveles educativos, sincronizando así con una preocupación internacional por la educación. Forma parte de ese proceso, que el gobierno del quinquenio pasado ha propuesto la Beca "Vocación de Maestro" (Pronabec, 2014, pág. 12), que constituye un esfuerzo para revertir el problema de la formación docente; exigiendo que acrediten tener quince (15) de promedio general en secundaria, lo que ha generado que en muchas regiones del país no haya postulantes. Ante esa realidad, nos hemos planteado investigar la relación existente entre la vocación profesional y la motivación por el estudio de los alumnos de educación, en la Universidad Nacional Toribio Rodríguez de Mendoza de Amazonas, en sus diferentes sedes.

Navarrete (2007, pág. 61), en su investigación sobre la construcción de una identidad profesional de los pedagogos de la Universidad Nacional Autónoma de México y de la Universidad Veracruzana, concluye en lo siguiente: durante el estudio sobre la construcción de la identidad profesional del pedagogo fue posible distinguir dos tipos: el escolar, cuya tarea se circunscribe al ámbito institucional de la escuela, y el social, que va más allá de los espacios escolares "formales" manteniendo un contacto más cercano con la comunidad y grupos sociales. El pedagogo general, de los sesenta y los noventa, goza de la ventaja de tener una formación disciplinar amplia y diversa, lo que le permite una visión de lo educativo y de lo escolar más o menos integral, tal vez suficiente si lo que se quiere de él es un consultor orientador sobre los problemas concretos y cotidianos que afectan los procesos de la educación y la escuela.

Lorenzo, Rojas y Cepero (2009), hizo una investigación sobre las preferencias profesionales y vocacionales del alumnado de secundaria y formación profesional específica. Desarrollado en la Facultad de Ciencias de la Educación de la Universidad de Granada. Las conclusiones siguientes: los motivos principales de elección para realizar los estudios de postsecundaria son el gusto por la profesión y las perspectivas del buen salario y empleo. Los ingresos económicos familiares y la probabilidad de aprobar influyen directamente en la opción de asistir a estudiar en la universidad.

Avendaño, González y Rodrigo (2012) investigaron los motivos para ingresar a las Carreras de pedagogía de los estudiantes del primer año de la Universidad de Concepción. Planteándose como objetivo, describir las motivaciones de los estudiantes de primer año de diferentes especialidades de Pedagogía para realizar estudios superiores en estas carreras. Concluyeron en: Un $23 \%$ de estudiantes tienen entre 21 y 31 años de edad, lo que implica hipotetizar que, al menos, casi un cuarto de los estudiantes del primer año de pedagogía no ingresa en forma directa a su actual carrera al salir de cuarto medio. Además, la mayoría de la muestra son mujeres $(67 \%) \mathrm{y}$, por lo mismo, el ingreso a casi todas las pedagogías de la Universidad de Concepción aparece feminizado, porque ingresan en forma exclusiva mujeres, sobre todo para educación parvularia.

Angulo (2008). Desarrolló la investigación: relación de la motivación y satisfacción con la profesión elegida con el rendimiento de los estudiantes de la Facultad de Educación de la UNMSM. El objetivo fue determinar la relación de la motivación y satisfacción con la profesión elegida con el rendimiento de estudiantes de educación, trabajaron con una muestra de 240 estudiantes de la Facultad de Educación, siendo los resultados: La motivación para el estudio de educación y la satisfacción con la profesión elegida están relacionados significativamente con el rendimiento académico de los estudiantes de la Facultad de educación. La motivación intrínseca no está relacionada significativamente con el rendimiento académico de 
los estudiantes de la facultad de educación y la motivación extrínseca sí está relacionada.

La vocación profesional es el proceso permanente en la que una persona evalúa e integra sus motivos, intereses y aptitudes con el fin de elegir aquella profesión que mejor se adecúe a su identidad personal. Existen teorías de la vocación profesional, como la de O.Crites (1984, pág. 42), quien subdivide en teorías no psicológicas, psicológicas y generales. Montero (2000, pág. 92) refiere que "la elección de la carrera profesional es: un asunto personal, asunto de jóvenes, asunto de familia, un privilegio, asunto de flexibilidad institucional y laboral, resultado de un test y asunto de género".

La vocación, según Ander-Egg (2005, pág. 189), es la inclinación, predisposición y afición predominante para un determinado tipo de estudios o profesión. Rimada, (2009, pág. 68), plantea principios rectores de la vocación profesional, relacionados con el modelo cognitivo de orientación, y son: principio de congruencia, principio de realidad, c) principio de la semejanza, d) principio residual, e) principio de aleación.

La motivación por el estudio, para Gonzáles (2008, pág. 31) constituye todo proceso psíquico (percepción, memoria, pensamiento, tendencia, emoción, sentimiento) que refleja la interacción establecida entre el sujeto y el mundo, y sirve para regular la actividad del individuo. Mientras que Santos (1990), citado por Polanco (2005, pág. 58), lo define como el grado en que los alumnos se esfuerzan para conseguir metas académicas que perciben como útiles y significativas. Existen muchos estudios sobre la motivación, asociados a la psicología, tal es así que, Gracián (2012, pág. 40), sistematiza las principales teorías modernas sobre la motivación en cuatro grandes escuelas: i) la psicoanalítica, organizada por Freud, ii) la conductista de Watson y Skinner. iii) la humanista, cuyo representante es Maslow, iv) la cognitiva de Piaget. Por su parte, Pintrich, (1997), Zimmerman y Kintasas, (1997), citado por De la Fuente, (2004, pág. 68), hablan sobre la teoría clásica de la orientación de metas, sosteniendo que existen metas académicas y sociales.

La hipótesis que nos planteamos es que la relación entre vocación profesional y motivación por el estudio es media.

\section{METODOLOGÍA}

Tipo y diseño de la investigación, el tipo de investigación es descriptiva y el diseño, descriptivo correlacional, de acuerdo con Hernández, Fernández y Baptista (2010).

Población: 666 estudiantes de las escuelas profesionales de educación intercultural bilingüe y de educación clásica de la Universidad Nacional Toribio Rodríguez de Mendoza de Amazonas en todas las Sedes.

Muestra: estuvo conformada por 243 estudiantes de las diferentes especialidades de educación intercultural bilingüe y educación.

Métodos y técnicas: la investigación se inició en febrero del año 2015, cuando en forma continua se observó el decremento progresivo de alumnos interesados en estudiar educación.

El instrumento fue un cuestionario llamado CUVOPROVE, en escala Likert, con 10 preguntas para medir la vocación profesional y 10 preguntas orientadas a mensurar la motivación por el estudio, con cinco alternativas, que van desde totalmente de acuerdo hasta totalmente en desacuerdo.

Análisis Estadístico: se realizó mediante el coeficiente de correlación, se calculó el coeficiente de correlación de Pearson usando el paquete estadístico SPSS, los resultados muestran una correlación bilateral altamente significativa entre las variables vocación profesional y motivación por el estudio, tal como se aprecia en el cuadro siguiente:

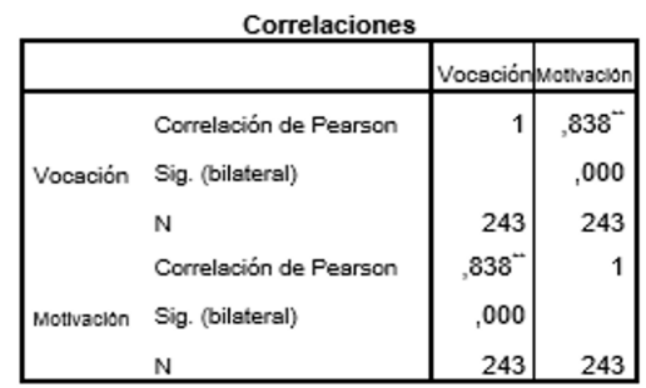




\section{RESULTADOS}

Tabla $\mathbf{N}^{\circ} 01$. Relación entre vocación profesional y motivación por el estudio del alumno de la Untrm.

\begin{tabular}{|c|c|c|c|c|}
\hline \multirow{2}{*}{ Alternativas } & \multicolumn{2}{|c|}{ Vocación profesional } & \multicolumn{2}{|c|}{ Motivación por el estudio } \\
\hline & f & $\%$ & $\mathbf{f}$ & $\%$ \\
\hline Totalmente de acuerdo & 73 & 30 & 68 & 28 \\
\hline De acuerdo & 48 & 20 & 47 & 19 \\
\hline Indeciso & 27 & 11 & 31 & 13 \\
\hline En desacuerdo & 56 & 23 & 61 & 25 \\
\hline Totalmente en desacuerdo & 39 & 16 & 36 & 15 \\
\hline Total & 243 & 100 & 243 & 100 \\
\hline
\end{tabular}

Fuente. Cuestionario administrado a los estudiantes universitarios

En la tabla $\mathrm{N}^{\circ} 01$, se aprecia la vocación profesional relacionado a la motivación por el estudio, de acuerdo al siguiente detalle: el 50\% de alumnos tienen vocación profesional para educador, mientras que un 39\% no lo tiene, así mismo el 11\% dice estar indeciso. En la motivación por el estudio, el 47\% se encuentran motivados, existiendo un empate estadístico con la vocación profesional; se concluye en que tienen una indecisión por sus estudios, constituyendo un probable motivo para abandonar la carrera en cualquier momento.

Tabla $\mathbf{N}^{\circ}$ 02. Relación entre vocación profesional y motivación por el estudio del alumno de educación de la Universidad Nacional Toribio Rodríguez de Mendoza de Amazonas, por sexo.

\begin{tabular}{cccccccccc}
\hline \multirow{2}{*}{ Alternativas } & \multicolumn{3}{c}{ Vocación profesional } & \multicolumn{3}{c}{ Motivación por el estudio } \\
& \multicolumn{2}{c}{ Mujeres } & \multicolumn{2}{c}{ Varones } & \multicolumn{2}{c}{ Mujeres } & \multicolumn{2}{c}{ Varones } \\
& $\mathrm{f}$ & $\%$ & $\mathrm{f}$ & $\%$ & $\mathrm{f}$ & $\%$ & $\mathrm{f}$ & $\%$ \\
\hline Totalmente de acuerdo & 22 & 29 & 51 & 30 & 20 & 27 & 48 & 29 \\
\hline De acuerdo & 15 & 20 & 33 & 20 & 14 & 19 & 33 & 20 \\
Indeciso & 15 & 20 & 12 & 7 & 16 & 21 & 15 & 9 \\
En desacuerdo & 14 & 19 & 42 & 25 & 17 & 23 & 44 & 26 \\
\hline Totalmente en desacuerdo & 9 & 12 & 30 & 18 & 8 & 11 & 28 & 17 \\
Total & 75 & 100 & 168 & 100 & 75 & 100 & 168 & 100 \\
\hline
\end{tabular}

Fuente. Cuestionario administrado a los estudiantes universitarios

En la tabla $\mathrm{N}^{\circ} 02$, sobre la vocación profesional relacionado a la motivación por el estudio del alumno de educación en base al sexo, se aprecia que el 50 y $49 \%$ de estudiantes varones y mujeres respectivamente tienen vocación profesional con su carrera, habiendo una ligera ventaja a favor de los hombres. En lo que respecta a la motivación por el estudio, el 46\% de alumnas tienen motivación por el estudio y en el caso de los varones, el 49\% dice estar motivado, significando esto un empate estadístico.

Tabla $\mathbf{N}^{\circ}$ 03. Relación entre vocación profesional y motivación por el estudio del alumno de educación de la Universidad Nacional Toribio Rodríguez de Mendoza de Amazonas, por especialidad.

\begin{tabular}{|c|c|c|c|c|c|c|c|c|c|c|c|c|}
\hline \multirow{3}{*}{ Alternativas } & \multicolumn{6}{|c|}{ Vocación profesional } & \multicolumn{6}{|c|}{ Motivación por el estudio } \\
\hline & \multicolumn{2}{|c|}{ Inicial } & \multicolumn{2}{|c|}{ Primaria } & \multicolumn{2}{|c|}{ Secundaria } & \multicolumn{2}{|c|}{ Inicial } & \multicolumn{2}{|c|}{ Primaria } & \multicolumn{2}{|c|}{ Secundaria } \\
\hline & $\mathrm{f}$ & $\%$ & $\mathrm{f}$ & $\%$ & $\mathrm{f}$ & $\%$ & $\mathrm{f}$ & $\%$ & $\mathrm{f}$ & $\%$ & $\mathrm{f}$ & $\%$ \\
\hline $\begin{array}{l}\text { Totalmente de } \\
\text { acuerdo }\end{array}$ & 15 & 26 & 42 & 32 & 16 & 31 & 14 & 24 & 41 & 31 & 13 & 25 \\
\hline De acuerdo & 13 & 22 & 23 & 17 & 12 & 23 & 11 & 19 & 24 & 18 & 12 & 23 \\
\hline Indeciso & 9 & 16 & 11 & 8 & 7 & 13 & 11 & 19 & 11 & 8 & 9 & 17 \\
\hline En desacuerdo & 11 & 19 & 35 & 26 & 10 & 19 & 13 & 22 & 37 & 28 & 11 & 21 \\
\hline $\begin{array}{l}\text { Totalmente en } \\
\text { desacuerdo }\end{array}$ & 10 & 17 & 22 & 17 & 7 & 13 & 9 & 16 & 20 & 15 & 7 & 13 \\
\hline Total & 58 & 100 & 133 & 100 & 52 & 100 & 58 & 100 & 133 & 100 & 52 & 100 \\
\hline
\end{tabular}


En la tabla $\mathrm{N}^{\circ} 03$, sobre la vocación profesional relacionado a la motivación por el estudio del alumno de educación en base a la especialidad de estudios, se aprecia que el 48, 49 y 54\% de alumnos de las especialidades de educación inicial, primaria y secundaria, respectivamente tienen vocación por estudiar educación, igualmente se hay un empate estadístico. En lo referente a la motivación por el estudio, el 43, 49 y 48\% de alumnos de las especialidades de inicial, primaria y secundaria se encuentran motivados por el estudio en su carrera que están siguiendo; frente a un 38,43 y $34 \%$ que dice no estar motivado.

Tabla $\mathbf{N}^{\circ}$ 04. Relación entre vocación profesional y motivación por el estudio del alumno de educación de la Universidad Nacional Toribio Rodríguez de Mendoza de Amazonas, por escuela profesional.

\begin{tabular}{cccccccccr}
\hline & \multicolumn{3}{c}{ Vocación profesional } & \multicolumn{4}{c}{ Motivación por el estudio } \\
Alternativas & \multicolumn{2}{c}{ Educación clásica } & Educación bilingüe & \multicolumn{2}{c}{ Educación clásica } & \multicolumn{2}{c}{ Educación bilingüe } \\
& $\mathrm{f}$ & $\%$ & $\mathrm{f}$ & $\%$ & $\mathrm{f}$ & $\%$ & $\mathrm{f}$ & $\%$ \\
\hline Totalmente de acuerdo & 6 & 20 & 67 & 31 & 4 & 13 & 64 & 30 \\
\hline De acuerdo & 4 & 13 & 44 & 21 & 3 & 10 & 44 & 21 \\
Indeciso & 3 & 10 & 24 & 11 & 3 & 10 & 28 & 13 \\
\hline En desacuerdo & 9 & 30 & 47 & 22 & 10 & 33 & 51 & 24 \\
Totalmente en desacuerdo & 8 & 27 & 31 & 15 & 10 & 33 & 26 & 12 \\
\hline Total & 30 & 100 & 213 & 100 & 30 & 100 & 213 & 100 \\
\hline
\end{tabular}

Fuente. Cuestionario administrado a los estudiantes universitarios

En la tabla $\mathrm{N}^{\circ} 04$, sobre la vocación profesional relacionado a la motivación por el estudio del alumno de educación, teniendo en cuenta la escuela profesional de educación clásica y educación intercultural bilingüe, se aprecia que solamente 10 estudiantes de la escuela profesional de educación clásica, tienen vocación por el estudio, frente a un 57\% que no la tiene; mientras que el 52\% de los alumnos de educación intercultural bilingüe dice tener vocación por sus estudios, y solamente un 37\% refiere no tenerla. En cuanto a la motivación por el estudio, solamente $23 \%$ de estudiantes de la escuela profesional de educación, se encuentran motivados por el estudio en la escuela profesional de educación clásica, frente a un 51\% de la escuela profesional de educación intercultural bilingüe que dice encontrarse motivado para el estudio.

En la figura $\mathrm{N}^{\circ} 01$, se aprecia la correlación entre vocación profesional y motivación por el estudio del alumno de la escuela profesional de educación clásica e intercultural bilingüe.

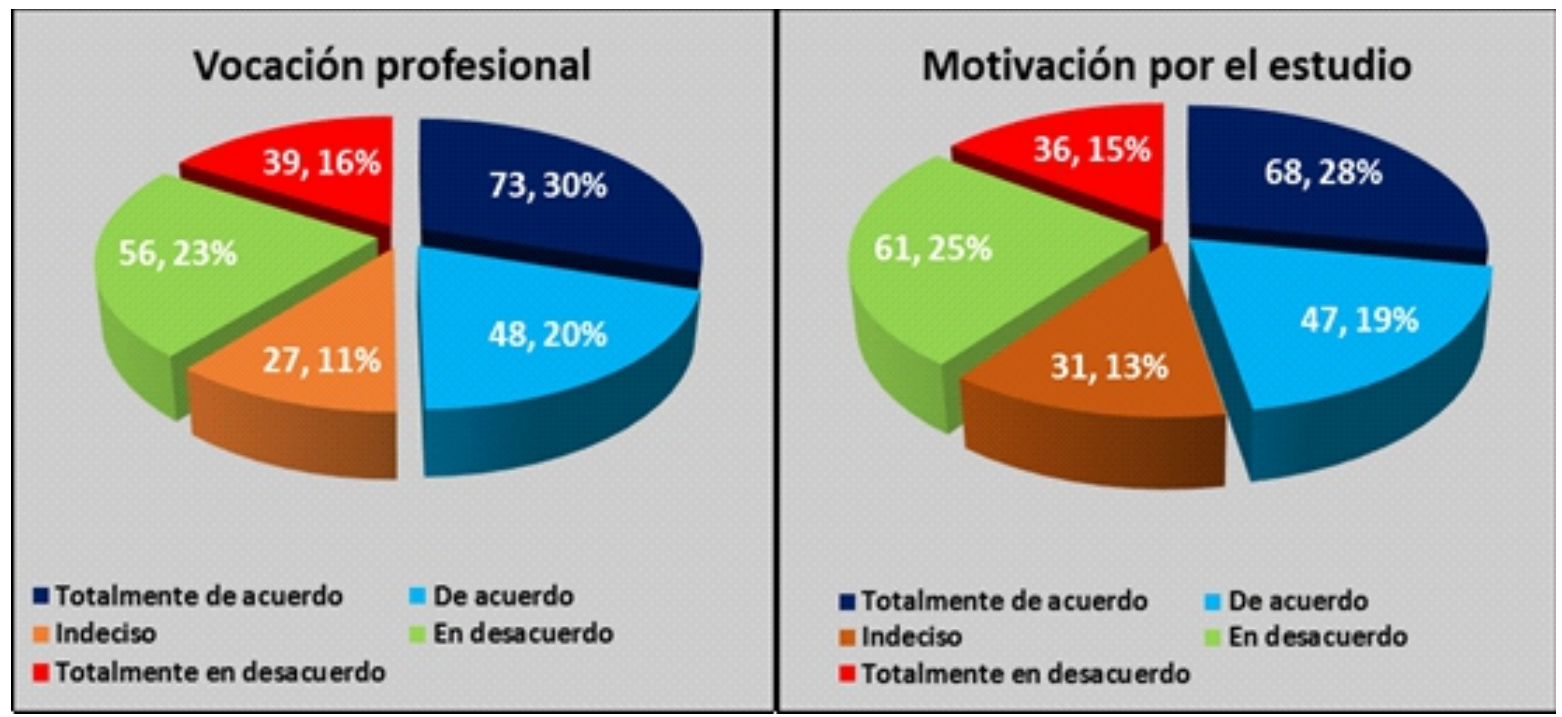

Fig. $\mathbf{N}^{\circ} \mathbf{0 1}$. Correlación entre vocación profesional y motivación por el estudio. 


\section{DISCUSIÓN}

Existe una correlación altamente significativa entre la vocación profesional y la motivación por el estudio del alumno de educación intercultural bilingüe y educación clásica, dado que el $50 \%$ dice tener vocación profesional, y el $47 \%$ dice estar motivado para el estudio. Resultados concordantes con el estudio de Angulo (2008), sobre la motivación y satisfacción con la profesión y rendimiento de estudiantes de la Facultad de Educación de la UNMSM, concluyendo que están relacionados significativamente sus variables de estudio.

La correlación entre la vocación profesional y la motivación por el estudio en cuanto a género, es directa alta, dado que, el 49 y $46 \%$ de mujeres responden estar de acuerdo y totalmente de acuerdo con sus estudios, en relación a la vocacional profesional y motivación por estudiar, respectivamente; en el caso de los varones, el 50\% dice estar de acuerdo y muy de acuerdo en la vocación profesional, y el $49 \%$ de ellos responde estar de acuerdo y muy de acuerdo en motivación por el estudio. Sin embargo, en promedio, un 20 y $16 \%$ de mujeres, dicen estar indecisos en su vocación profesional y motivación por el estudio, este alto porcentaje se debe al predominio del machismo presente en las comunidades nativas awajun y wampis, donde funciona la sede de la escuela de educación intercultural bilingüe y alberga al 90\% de estudiantes que participaron en el estudio. Los resultados también muestran una ligera ventaja de los varones en términos de preferencia por la carrera. En términos comparativos con los hallazgos de Avendaño, Gonzáles y Rodrigo (2012), sobre "Motivos para ingresar a las carreras de pedagogía en primer año de la Universidad de Concepción", existe divergencia, dado que el $67 \%$ de mujeres estudian educación. Por otro lado, se aprecian coincidencias con el trabajo de Navarrete (2010), sobre "Construcción de una identidad profesional de los pedagogos de la Universidad Nacional Autónoma de México y de la Universidad de Veracruz", sobre todo en las sedes de educación intercultural bilingüe, dado que el docente varón además de su desempeño profesional es el líder de su comunidad, siendo el gran referente para los jóvenes que al final por cuestiones de identidad interpersonal se identifican y se inclinan por la profesión docente.

La correlación entre vocación profesional y motivación por el estudio, en lo que respecta a la escuela profesional, permite observar que el $57 \%$ de estudiantes de la escuela profesional de educación, dice estar en desacuerdo y totalmente en desacuerdo con su vocación para estudiar educación, incrementándose el porcentaje en estas alternativas, en 9 puntos en relación a la motivación por el estudio. Mientras que en la escuela profesional de educación intercultural bilingüe, más del 50\% de estudiantes dice tener vocación profesional y estar motivados por sus estudios en educación.

\section{CONCLUSIONES}

1.La correlación entre vocación profesional y motivación por el estudio de los alumnos de la escuela profesional de educación intercultural bilingüe y escuela profesional de educación, es altamente significativa, con 0,838 bilateral, según la prueba Pearson.

2.Existe una correlación directa alta entre la vocación profesional y la motivación por el estudio del alumno de educación, dado que un $50 \%$ de estudiantes dice tener vocación profesional y un $47 \%$ dice estar motivado para el estudio.

3.La correlación entre la vocación profesional y la motivación por el estudio en relación al género, es directa alta, dado que, el 49 y $46 \%$ de mujeres responden estar de acuerdo con sus estudios de educación, en vocación profesional y motivación por el estudio respectivamente, y en el caso de los varones, el $50 \%$ dice estar de acuerdo y muy de acuerdo en vocación profesional, y el $49 \%$ está de acuerdo y muy de acuerdo en motivación por el estudio.

4.La correlación entre la vocación profesional y la motivación por el estudio en relación a la especialidad, es directa alta, por cuanto, el 48, 49 y $54 \%$ dice estar de acuerdo y totalmente de acuerdo con sus estudios de educación, en inicial, primaria y secundaria; y en cuanto a la motivación por el estudio, dicen estar de acuerdo y muy de acuerdo, el 43,49 y $48 \%$ en la especialidad de inicial primaria y secundaria, respectivamente.

5.La correlación entre vocación profesional y motivación por el estudio en lo que respecta a la variable escuela profesional, un elevado $57 \%$ de estudiantes de la escuela de educación, dice estar en desacuerdo y totalmente en desacuerdo con su vocación para estudiar educación, incrementándose el porcentaje en 9 puntos en relación a la desmotivación por el estudio; mientras que en la escuela profesional de educación intercultural bilingüe están de acuerdo y muy de acuerdo, en vocación y motivación por sus estudios, más del $50 \%$ de alumnos.

\section{REFERENCIAS BIBLIOGRÁFICAS}

Ayzanoa, G. (2003). La carrera pública magisterial. Lima.

Angulo, J. (2008). Relación de la motivación y satisfacción con la profesión elegida con el rendimiento de los estudiantes de la Facultad de Educación de la UNMSM. (tesis de pregrado). UNMSM. Perú. 
Ander-Egg, E. (2005). Diccionario pedagógico. Buenos Aires: Magisterio.

Avendaño, C. y González, R. y Rodrigo (2012). Motivos para ingresar a las carreras de pedagogía de los estudiantes del primer año de la Universidad de Concepción (tesis de pregrado). Universidad de Concepción. Chile.

Delors, J. (1999). La educación encierra un tesoro. Madrid. Alfaomega.

De la Fuente, J. (2004). Perspectivas recientes en el estudio de la motivación: la teoría de la orientación de meta. (Tesis de pregrado). Universidad de Almeria. España.

Díaz, J. y Martins, A. (1982). Orientaciones didácticas para la docencia. San José de Costa Rica: IICA.

Gracián, B. (2012). Teorías sobre la motivación. Boletín Unidad de conocimiento. Funfació factor huma.

Gonzáles, D. (2008). Psicología de la motivación. La Habana: ECEMED.

Hernández, Fernández y Baptista. (2010). Metodología de la investigación cientifica. Ciudad de México: McGraw-Hill.

Lorenzo, M. Rojas, F. y Cepero Del M. (2009), Tesis: las perspectivas profesionales y vocacional del alumnado de secundaria y formación profesional específica (tesis de pregrado). Universidad de Granada.

Navarrete (2010). Construcción de una identidad profesional de los pedagogos de la Universidad Nacional Autónoma de México y de la Universidad Veracruzana (tesis de pregrado). Universidad autónoma de México.

Ministerio de Educación (2014). Guía de orientación de estudios. Secretaría Nacional de la Juventud. Lima. Visitado el 19 de febrero de 2016 . D i s p o n i b l e e n : file://C:/Users/cristian/Downloads/guia_de_o rientacion_de_estudios.pdf.

Montero, M. (2000). Elección de carrera profesional: Visiones, promesas y desafios. Ciudad de Juárez. Imprenta de la Universidad Autónoma de Juárez.

Pronabec (2014). Programa Nacional de Becas, Boletín Informativo, Lima.
Román, M. (2011). Aprender a aprender en la sociedad del Conocimiento. Madrid: Conocimiento S.A.

Polanco, A. (2005). La motivación en los estudiantes universitarios. Revista electrónica "Actualidades investigativas en educación". Universidad de Costa Rica. Redalyc.org.

Rimada, B. (2009). Manual de orientación profesional universitaria. Ciudad de México: Trillas. 